\section{L'Actualité économique}

L'ACTUALITÉ ÉCONOMIQUE

\section{Évolution économique du Québec 1950-1965}

\section{Marcel Daneau}

Volume 41, numéro 4, janvier-mars 1966

URI : https://id.erudit.org/iderudit/1003128ar

DOI : https://doi.org/10.7202/1003128ar

Aller au sommaire du numéro

Éditeur(s)

HEC Montréal

ISSN

0001-771X (imprimé)

1710-3991 (numérique)

Découvrir la revue

Citer cet article

Daneau, M. (1966). Évolution économique du Québec 1950-1965. L'Actualité

économique, 41(4), 659-692. https://doi.org/10.7202/1003128ar
Ce document est protégé par la loi sur le droit d'auteur. L'utilisation des services d'Érudit (y compris la reproduction) est assujettie à sa politique d'utilisation que vous pouvez consulter en ligne.

https://apropos.erudit.org/fr/usagers/politique-dutilisation/ 


\title{
Évolution économique du Québec 1950-1965
}

\begin{abstract}
Si l'économie de la province de 2uébec a suivi, au cours des dernières années, le mouvement général d'expansion cyclique qui a prévalu en Amérique du Nord, elle a connu, en même temps, des modifications structurelles importantes sans parvenir à éliminer pour autant un certain nombre de problèmes qui s'y posent de façon chronique. De plus, la législation visant directement ou indirectement l'activité économique a été particulièrement importante depuis quatre ou cinq ans. Enfin, pour des raisons qui ne tiennent pas toutes à l'économie, la croissance de la Province a été suivie, ces derniers temps, avec une attention nouvelle, C'est pourquoi nous pensons que l'article qui suit, pourra en intéresser plusieurs. In ne prétend pas élucider une question particulière mais constitue un dossier auquel plusieurs pourront sans doute référer avec intérêt.
\end{abstract}

N. D. L. R.

De profondes transformations ont bouleversé l'économie du Québec au cours des quinze dernières années. Et tous les secteurs de l'activité économique en furent touchés. Certains ont connu une expansion rapide, d'autres, lente. La production, la main-d'œuvre, les prix et les investissements sectoriels reflètent des tendances différentes. Le secteur primaire, par exemple, a une tenue et un déve' loppement économiques moins bons que ceux du secondaire et du tertiaire. D'une façon similaire, les régions économiques du Québec subirent aussi de grands changements et dans l'ensemble, les régions périphériques en profitent moins que les régions concentriques. De même, l'importance relative des agents économiques se modifie. L'Etat provincial joue un rôle de plus en plus grand dans l'activité économique globale et sectorielle en déplaçant, en supportant, en lançant des entreprises qui, autrement, n'auraient jamais vu le jour ou auraient été menacées de disparaître. Bref, la « révolution tran- 


\section{L'ACTUALITE ECONOMIQUE}

quille » a pris durant cette période les aspects d'une révolution économique et industrielle. Comme toute révolution, celle'ci change la face des choses et des gens. L'adaptation est parfois douloureuse. Elle change des structures et des mentalités qui peuvent être souples ou rigides. Elle engendre le développement et la friction. C'est un phénomène normal car la rapidité d'adaptation des individus est variable. Certains s'y opposent, d'autres l'acceptent ou l'appuient. C'est ainsi que se forme une nation sur le plan socio économique. Globalement, les multiples facettes économiques du Québec se résument aux principaux points suivants durant cette période. 1) La main'd'œuvre s'est accrue considérablement en lais' sant subsister un chômage chronique. 2) Le taux d'augmentation du revenu personnel s'est accéléré durant les périodes d'activité économique intense pour ensuite diminuer lors des dépressions quasicycliques. 3) Les investissements, l'une des composantes les plus sensibles de la comptabilité nationale, ont été fort influencés par le comportement combiné de l'entreprise privée et de l'Etat. 4) L'Etat lui-même acquiert une proportion de plus en plus importante du revenu global et son action fiscale prend des dimensions encore insoupçonnées dans tous les domaines. 5) Le relèvement de l'économie québécoise est largement conditionné par sa capacité de concurrence sur les marchés intérieurs et extérieurs. Ayant une économie ouverte, et qui est en passe de le devenir de plus en plus, le Québec ne pourra soutenir la concurrence, se développer et survivre que s'il fait montre d'un dynamisme constant et soutenu sur tous les marchés. 6). La formation d'entrepreneurs canadiens français compétents, possédant le sens des affaires et de l'innovation, a pris un sens aigu d'actualité. 7) Le Québec s'est forgé ou se forge les outils financiers nécessaires à son expansion. 8) Les faiblesses inhérentes à certaines régions se sont accentuées. 9) $\mathrm{La}$ position relative des secteurs économiques, les uns par rapport aux autres, s'est complètement transformée.

Reprenons chacun de ces points, à l'échelle provinciale d'abord, pour les analyser, ensuite, par secteur et par région.

Pour mesurer la croissance économique d'un pays, on utilise ordinairement le taux de croissance à long terme du volume de 


\section{EVOLUTION ECONOMIQUE DU QUEBBEC}

production totale. Or, au Québec, cette comptabilité globale n'existe pas. Nous emploierons done d'autres variables pouvant servir d'in' dices de croissance.

Lorsque les différents revenus reçus par les citoyens du Québec sont mesurés de 1950 à 1963, il s'en dégage les faits suivants :

- le revenu personnel total a plus que doublé ;

- le revenu personnel par habitant a augmenté de 80 pour cent ;

- le revenu personnel disponible est passé de 3.15 à 7.54 milliards de dollars ;

- les traitements, salaires et revenus supplémentaires de la main. d'cuvre ont plus que doublé ;

- le revenu net des agriculteurs provenant de l'exploitation agricole a diminué de 14 pour cent;

- le revenu net des entreprises individuelles non agricoles s'est accru de 88 pour cent ;

- les intérêts, dividendes et loyers nets des personnes n'ont pas tout à fait triplé ;

- les transferts des gouvernements, à l'exclusion de l'intérêt sur la dette publique ont plus que quadruplé.

Le taux de croissance annuel moyen de ces différents revenus varie entre 5 et 8 pour cent, sauf celui des transferts gouvernementaux qui atteint 11 pour cent. En agriculture, le taux de croissance est négatif, soit une diminution moyenne de 1 pour cent.

Bien entendu, le taux de croissance a évolué de façon irrégulière durant cette période. La croissance économique du Québec est marquée par des changements brusques d'orientation, soit vers la baisse, soit vers la hausse. Des hausses remarquables eurent lieu en 1950-1951, et 1955-1956. D'autre part, des baisses tout aussi remarquables furent enregistrées en 1953-1954 et 1958-1959. Depuis 1960 , l'essor économique du Québec est croissant et soutenu. Il suit ainsi la tendance nord-américaine de ces dernières années. Des flé, chissements sont cependant prévisibles, attribuables à des variations cycliques à court terme. Si l'évolution économique des vingt dernières années se répétait, un certain ralentissement des affaires n'est pas exclu durant les deux prochaines années. Car l'économie québécoise, comme toute autre économie d'ailleurs, engendre durant sa phase de croissance des forces qui amèneront un revirement ou une phase de décroissance. L'apparition d'une telle phase est une certi: 
tude, seul le moment de son apparition ne l'est pas. Quoi qu'il en soit, un examen attentif des tendances de l'économie du Québec au cours de la dernière décennie nous révèle que la production globale et les revenus ont augmenté plus rapidement au Québec qu'au Canada et que le bien-être de la population québécoise s'est amélioré sensiblement dans l'ensemble. Mais le Québec n'a pu extirper pour autant la plaie du chômage.

De 1945 à 1953, l'emploi de la main-d'œuvre au Québec s'est maintenu à un haut niveau grâce à une forte demande de biens et services sur les marchés intérieurs et extérieurs. La satisfaction des besoins accumulés durant la dépression de 1930 et des années de guerre explique l'expansion rapide de l'économie québécoise de cette période. Mais le retour à des conditions de paix après la guerre de Corée et le relèvement économique de l'Europe atténuèrent les forces expansionnistes ou réduisirent la demande de notre production et ce ralentissement eut des répercussions immédiates sur l'emploi au Québec. A partir de 1954, la proportion de la main-d'œuvre en chômage par rapport à la main-d'œuvre totale n'a cessé de croitre jusqu'en 1961. Même les périodes d'expansion rapide comme celles de 1955 et 1956 n'ont pu réduire le chômage à un niveau acceptable, soit environ 3 pour cent. En fait, de 1950 à 1953, le taux moyen de chômage au Québec a été de 3.7 pour cent. De 1954 à 1957, il a augmenté de moitié pour atteindre une moyenne de 5.8 pour cent, puis, de 1958 à 1961, il s'est encore accru de 50 pour cent pour se fixer à 8.8 pour cent. De 1962 à 1964, le taux de chômage a légèrement fléchi à 7.2 pour cent. Il dépasse encore largement les meilleures années d'expansion économique des deux dernières décennies.

L'incidence du chômage au Québec est très inégale. Elle touche toute l'économie, il est vrai, mais certaines régions (Gaspésie, Bas St-Laurent, Abitibi), certaines occupations (agriculture, pêche, fo rêt, construction) et certains groupes d'âge (main-d'œuvre jeune non spécialisée et les plus âgés) en sont frappés plus que d'autres.

A l'échelle régionale, en particulier, le taux de chômage atteint durant certaines périodes de l'année des sommets inimaginables. En Gaspésie, par exemple, 62.5 pour cent des pères de famille d'âge actif tombent en chômage durant la période hivernale. En été, ce 
taux est réduit à 17.5 pour cent ${ }^{1}$. Les pertes de ressources humaines et matérielles qui découlent d'une telle situation, tant à l'échelle provinciale que régionale, sont incalculables. Et, à moins que des politiques expansionnistes continues ne soient mises en place, une détérioration nouvelle du marché du travail ne peut être écartée. Une analyse de l'évolution de la main-d'œuvre au Québec révèle les caractéristiques suivantes, de 1950 à 1964 :

- la main-d'œuvre totale a augmenté de 35 p.c.;

- la main-d'œuvre non agricole s'est accrue de 50 p.c.;

- la main-d'œuvre agricole a diminué de moitié ou à peu près ;

- la main'd'ouvre en chômage a plus que doublé, passant de 63,000 en 1950 à 131,000 en 1964 .

Durant les quinze dernières années, le taux moyen annuel d'accroissement de la main-d'œuvre en chômage atteint 8.4 pour cent. Si un tel taux se maintient encore dix ans, le nombre de chômeurs doublera presque d'ici à 1974, pour atteindre le chiffre respectable d'environ 240,000 chômeurs. D'autre part, le Québec semble au seuil d'une augmentation de la main-d'œuvre totale sans précédent dans le passé. La haute natalité des années de guerre et de l'après. guerre fera déferler sous peu une vague de jeunes sur le marché du travail. De 1950 à 1964, l'augmentation de la main-d'œuvre totale a été de 503,000 , soit un accroissement annuel moyen d'en. viron 38,000 individus ou un taux moyen d'à peu près 2.2 pour cent. De 1964 à 1974, le taux annuel moyen d'accroissement de la main d'œuvre atteindrait 3.1 pour cent. $\hat{A}$ ce rythme, il faudra créer un minimum de 630,000 emplois durant la prochaine décennie. Bref, pour éviter de graves problèmes socióéconomiques, il faudra accélérer la croissance économique du Québec au cours des prochaines années. Et, pour ce faire, il ne faut compter en aucune façon, sur le secteur agricole dont le taux de diminution annuel moyen a été de 4.9 pour cent de 1950 à 1964 . Enfin, nous tenons à signaler un autre point en ce qui a trait à l'emploi. Certains groupes de travailleurs ont été sans emploi durant de longues périodes. Dans les cas extrêmes, une telle prolongation a rendu cette main-d'œuvre pratiquement inemployable. $\AA$ une croissance écono mique élevée devra donc correspondre une réadaptation de cette

r. Daneau, Marcel, Situation économique des familles rurales de la Gaspésie, $196_{3}$ (inédit). 
main-d'œuvre, permettant ainsi d'atténuer au minimum les conséquences des changements structurels dans certains secteurs de notre économie.

On ne peut parler de l'évolution économique du Québec sans mentionner l'une de ses composantes les plus importantes : les finances publiques. De 1950 à 1963, les gouvernements provincial et municipaux ont absorbé une proportion de plus en plus grande de la production globale au Québec. Cette proportion atteindrait, dans les années récentes, environ 32 pour cent de notre production. C'est donc dire que l'apport gouvernemental à l'économie québécoise est devenu primordial. Bien plus, durant la dernière décennie, le taux d'accroissement des dépenses gouvernementales a nettement dépassé celui de la production provinciale. L'augmentation s'est surtout manifestée par suite d'un accroissement marqué du volume et du coût des divers services publics, comme l'enseignement, les routes, la santé et le bien-être social. Toutefois, cette évolution a été extrế mement irrégulière. De 1950 à 1963, elle peut être divisée en deux phases : la première couvre la période de 1950 à 1960 , la deuxième, de 1960 à nos jours.

Durant la première phase, le taux d'accroissement annuel moyen des dépenses totales générales brutes provinciales a été de 10 pour cent environ. Celui des dépenses totales municipales atteignait un peu plus de 9 pour cent. La vitesse de changement était à peu près similaire à ces deux paliers gouvernementaux. Les dépenses provin. ciales étaient une fois et demie plus grandes que les dépenses municipales.

Durant la deuxième phase, le taux d'accroissement des dépenses provinciales double, alors que le taux d'accroissement des dépenses municipales diminue de moitié. Dans une certaine mesure, il y a déplacement des recettes et des dépenses des municipalités vers le gouvernement provincial. Une nouvelle répartition des responsabilités entre les gouvernements provincial et municipaux est présentement en cours et provoque de profonds changements et de continuelles adaptations. Si cette tendance se continue, comme il est à prévoir, le déplacement des recettes fiscales produira de fortes tensions sur les finances municipales qui ne peuvent jouer que sur un champ très restreint de l'assiette fiscale. 
Le Québec a connu, de 1950 à 1964, une période d'investissements privés et publics sans précédent. De nombreux facteurs ont contribué à cette évolution. L'augmentation de la population et des familles a accru la demande de nouvelles habitations. La mise en valeur de nos ressources naturelles a exigé des capitaux massifs. Des revenus plus élevés en général ont stimulé la demande de biens et services et des biens d'équipement qui les produisent. La croissance urbaine a eu d'importantes répercussions sur les dépenses d'infrastructure ou d'équipement social, comme les routes, les aqueducs et les écoles. En 1961, par exemple, 77 pour cent des ménages vivaient en milieu urbain. De façon générale, les conditions de financement sont demeurées faciles grâce à des taux d'intérêt relativement bas. Une expansion rapide des investissements s'est donc produite au Québec au cours des quinze dernières années et nous pouvons en dégager les traits suivants. De 1950 à 1964, les investissements dans la construction, la machinerie et l'équipement ont plus que triplé. Le taux d'accroissement annuel moyen a été de 8.9 pour cent. Dans l'ensemble, le taux d'accroissement de la construction a été plus élevé que celui de la machinerie et de l'équipement. Ce qui frappe surtout lorsque l'on examine attentivement le comportement des investissements, c'est leur relative instabilité durant cette période. Non seulement le taux d'accroissement variet il d'une année à l'autre, mais encore passe-til par des phases successives d'expansion et de contraction. Point encore plus important, son comportement erratique conditionne fortement léconomie générale du Québec. Un accroissement des investissements se traduit par une expansion de l'économie québécoise et vice versa. De 1950 à 1954 et de 1956 à 1960, le taux d'accroissement des investissements décroît. De 1954 à 1956 et de 1960 à 1964, il croît. Parallèlement, l'économie du Québec suit les mêmes tendances : le chômage ou l'emploi se généralisent, les revenus s'accroissent ou s'affaissent. Depuis quelques années, cependant, l'amplitude des variations s'atténue grâce à l'ap' port stabilisateur des politiques fiscales des gouvernements fédéral et provincial. Des investissements croissants dans le secteur public équilibrent, en partie, les variations qui apparaissent dans le secteur privé. Du point de vue du rapport entre les investissements et la production, notons finalement que le Québec devance le Canada, les Etats-Unis et un grand nombre de pays européens. De fait, les 


\section{L'ACTUALITE ECONOMIQUE}

nouveaux investissements au Québec en pourcentage de la demande globale atteignaient environ 23.6 en 1964 . $\AA$ une demande globale estimée à 12 milliards de dollars, les investissements privés et publics contribuaient pour une somme de 2.7 milliards de dollars. Au Canada, ce pourcentage est d'environ de 18 et, aux Etats-Unis, de 12. De forts investissements expliquent d'ailleurs une croissance plus dynamique au Québec qu'au Canada.

L'épargne au Québec est-elle suffisante pour financer et maintenir un tel niveau d'investissements? Sinon, ne doiton pas importer des épargnes de l'extérieur du Québec? Une estimation de l'épargne nette des personnes et de l'épargne des entreprises les établit à moins d'un milliard et quart en 1961. Pour ce qui est de l'épargne gouvernementale, elle aurait été négative ces dernières années, les dépenses excédant les recettes. Par surcroît, les investissements de l'État ont été financés en grande partie au moyen d'em. prunts.

Si l'on compare l'épargne nette des personnes et celle des entreprises avec les immobilisations totales privées et publiques, on constate l'évolution suivante : en 1951, l'épargne atteint 80 pour cent des investissements; en 1953, 70 pour cent; en 1957, 49 pour cent ; en 1959 et 1961, 57 pour cent. Il y a une diminution relative des épargnes par rapport à l'accroissement des investissements. Les épargnes annuelles ne seraient pas suffisantes pour financer les investissements au Québec. La croissance économique y dépend donc encore dans une large mesure de l'entrée de capitaux en provenance des autres provinces canadiennes, des Etats-Unis ou de l'Europe. Ce qui nous amène à examiner l'évolution des dépenses de consommation.

De 1950 à 1963, les ventes au détail effectuées au Québec ont plus que doublé, passant de 2.2 à 4.8 milliards de dollars. Le taux d'accroissement annuel moyen a été de 6.2 pour cent durant cette période. Mais l'augmentation a varié selon les années d'expansion ou de contraction économiques, étant plus lente dans celles'ci que dans celles-là. De façon générale, cependant, une amélioration sen sible du niveau de vie moyen s'est traduite par une augmentation rapide de la demande de biens durables et non durables et de ser- 
vices. La demande de biens durables, comme l'automobile, a évolué très rapidement au cours des quinze dernières années. Par exemple, la valeur au détail de tous les véhicules automobiles neufs vendus au Québec a plus que triplé. De 167 millions en 1950, les ventes ont grimpé à 527 millions en 1963. Quel est maintenant l'effet d'un tel accroissement sur l'épargne personnelle? Le pourcentage du revenu personnel disponible absorbé par l'épargne nette des personnes varie d'une année à l'autre. En longue période, il se maintient en moyenne à 7 pour cent du revenu disponible. Si un tel taux d'épargne se maintient au cours des années à venir au Québec, les épargnes nettes globales personnelles augmenteront avec un accroissement du revenu disponible. Mais, sans une élévation du taux d'épargne luimême, ce qui est peu probable, les épargnes personnelles ne croîtront jamais assez rapidement pour permettre un financement substantiel des investissements au Québec. D’autre part, l'épargne forcée par l'établissement d'un système universel de caisse de retraite constituera d'ici peu une importante source de capitaux pour la province. Quelles en seront les répercussions sur le volume des épargnes et sur le taux des épargnes privées, nul ne saurait encore le dire. L'ex' périence américaine tend à laisser croire que les effets pourraient ne pas être très sensibles.

Une économie dynamique implique un rythme élevé de produc' tion dans tous les secteurs d'activité économique qui la composent : le progrès ne saurait être rapide sans un degré élevé d'utilisation de toutes les ressources. En somme, les tendances et les problèmes d'adaptation dans les industries primaires, secondaires et tertiaires conditionnent l'évolution économique du Québec. Nous examinerons donc maintenant l'économie du Québec par secteur.

Les industries primaires (agriculture, forêt, pêche, mines) jouent encore un rôle très important dans l'économie du Québec. Depuis quelques années, toutefois, elles font face à de réels problèmes d'adaptation. L'industrie agricole, en particulier, subit des changements économiques importants et parfois aussi extrêmement douloureux. 
Durant la période 1950-1963, le revenu brut total de l'agriculture a augmenté très lentement au Québec, soit en moyenne d'environ 2.6 pour cent par an. Cependant, en termes de valeur, la production agricole a baissé considérablement par rapport à la pro duction totale. En pourcentage du produit provincial brut, la pro duction de l'agriculture était rendue à environ 5 pour cent en 1963. $\AA$ une production totale estimée à 11 milliards de dollars, l'agriculture contribuait pour une valeur de 554 millions. L'évolution du revenu net total est encore plus frappante au cours des quinze dernières années. Le taux d'accroissement annuel moyen du revenu net total a été négatif : diminution moyenne de 0.5 pour cent. Cette diminution moyenne fut accompagnée par un très fort mouvement de population vers les régions urbaines. Le nombre de personnes employées dans le secteur agricole est passé de 254,000 en 1950 à 121,000 en 1964. Il en résulte que, dans l'ensemble, le revenu net par exploitant de ferme a eu tendance à augmenter durant cette période car, à une diminution relative du revenu, correspond une diminution plus que proportionnelle de la main-d'œuvre. Il n'en reste pas moins des différences substantielles entre les revenus des exploitants agricoles et de ceux qui travaillent dans d'autres secteurs de l'activité économique. Les éléments principaux de ces différences sont nombreux. Une baisse de la productivité agricole sur un très grand nombre de fermes du Québec en est un. Mais l'élément premier est une détérioration des termes d'échange de l'agriculture québécoise. Les prix des produits agricoles ont augmenté moins rapidement que ceux des produits industriels ou, ce qui est la même chose, les prix reçus par les cultivateurs à la vente des produits agricoles s'accroissent moins rapidement que les prix payés par les cultivateurs, à l'achat de produits industriels ou transformés. La cause fondamentale de la détérioration des termes d'échange est la croissance relativement lente de la demande des produits alimentaires par rapport à la croissance des revenus. Aujourd'hui, plus d'argent est aussi dépensé pour les services reliés aux denrées alimentaires que pour les denrées elles-mêmes. Parmi les autres facteurs qui ont pu contribuer au déclin relatif des revenus des cultivateurs du Québec, notons le manque d'instruction et de formation professionnelle d'un grand nombre d'entre eux, des ressources financières insuffisantes pour changer d'occupation ou pour agrandir 
leur exploitation agricole et, enfin, un certain vieillissement des agriculteurs depuis quelques années. Toute politique d'aide à l'agriculture au Québec qui aura pour objet le soutien des revenus des cultivateurs devra tenir compte de l'ensemble de ces éléments. Au trement, elle risque d'être très aléatoire.

L'industrie des pêches n'est pas aussi importante que les autres industries primaires. Néanmoins, elle occupe une place importante dans l'économie de certaines régions, comme les Iles-de-la-Madeleine, la Gaspésie et la basse Côte-Nord. La production totale des pêches québécoises a augmenté à un taux d'environ 2 pour cent par année durant les trente dernières années. Cette industrie dépend des marchés d'exportation pour environ 70 pour cent de sa production. De 1950 à 1963 , la valeur totale à l'expédition a doublé pour atteindre les dix millions de dollars. Quant aux immobilisations, elles ont presque doublé durant cette période grâce au financement par l'Etat de l'achat de bateaux et d'outillage par les pêcheurs. Le nombre de pêcheurs a eu tendance à se stabiliser à environ 6,000 depuis 1958 et ce nombre est encore considérable par rapport à la production. En conséquence, la productivité et les revenus sont faibles particulièrement au niveau de la pêche côtière. De plus, les pêcheurs ont eu à subir une détérioration de leurs termes d'échange tout comme les agriculteurs. Les prix reçus pour les produits de la pêche croissent moins vite que les prix payés pour les produits industriels. Certaines mesures gouvernementales destinées à relever le revenu des pêcheurs ont freiné leur mobilité tendant ainsi à perpétuer les problèmes qui se posent dans ce secteur. Bien que les conditions actuelles du marché soient excellentes, une concurrence très forte des producteurs des Provinces Maritimes et de certains pays euro péens sur les marchés extérieurs laisse peu d'espoir d'une réduction des difficultés chroniques de cette industrie québécoise.

La production de l'industrie minière a pris une expansion rapide depuis 1950 ; l'augmentation moyenne annuelle de la valeur totale de la production minérale a été d'environ 3 pour cent. De 1950 à 1963 , la valeur de la production minérale a plus que doublé, passant de 221 millions à 520 millions de dollars. Le taux d'accroissement semble toutefois moins rapide depuis 1957 que durant les années 
précédentes. Ce taux varie aussi d'une année à l'autre suivant la conjoncture économique ou la demande sur les marchés d'exportation. Il ne faut pas oublier qu'au moins 60 pour cent de notre production minérale trouve preneur à l'étranger. Au cours des quinze dernières années, l'industrie minière s'est signalée non seulement par une croissance rapide mais aussi par son déplacement vers les régions nordiques du Québec. L'exploitation du minerai de fer, en particulier, s'est développée d'une façon spectaculaire. Ce développe' ment s'est traduit par une forte augmentation des dépenses en immobilisations jusqu'en 1957 surtout. Par la suite, les investisse' ments ont plutôt eu tendance à se stabiliser. Le nombre d'employés dans les mines et carrières a aussi légèrement augmenté jusqu'en 1957 et il s'est maintenu ensuite à environ 32,000. La production par travailleur s'est accrue à un rythme très rapide durant cette période. Une utilisation intense de capital et l'importance grandissante des opérations mécanisées en sont les raisons fondamentales. Il s'ensuit que les salaires sont relativement élevés dans l'industrie minière mais l'embauche se limite de plus en plus à des travailleurs spécialisés et capables de voir au fonctionnement des machines. L'in dustrie minière, malgré son développement, offre donc un débouché très limité à la main'd'œuvre du Québec, et particulièrement à celle qui vit dans les régions défavorisées.

La valeur totale de la production forestière a augmenté en moyenne d'environ 7 pour cent par année de 1950 à 1960 pour atteindre finalement le milliard de dollars. Cette augmentation ne fut pas soutenue durant cette période à cause des changements de la demande sur les marchés d'exportation. La majeure partie de notre production de pâtes et papiers et de notre bois de construction étant exportée, il est très difficile, sinon impossible, de se soustraire aux variations de la demande et de la concurrence sur les marchés exté rieurs. L'industrie des pâtes et papiers est celle dont l'augmentation a été la plus sensible, soit 47 pour cent en dix ans. La production de l'industrie du bois de construction ne s'est accrue que de 14 pour cent durant la même période. Un changement considérable s'est produit dans l'emploi de la main d'œuvre forestière. L'introduction de la scie mécanique et de la machinerie lourde en forêt fait que le travail forestier qui se faisait pendant l'hiver se fait maintenant de 
plus en plus pendant l'été. Les exploitants forestiers tendent aussi à embaucher leur main-d'œuvre à l'année longue. Une tendance à la baisse de l'emploi dans les opérations forestières et dans les usines est donc apparue depuis quelques années. Ces changements peuvent avoir de profondes répercussions sur certaines catégories de maind'œuvre dont une partie des revenus provenait du travail en forêt, tels les cultivateurs et les pêcheurs. Dans certaines régions, cette évolution a donné une importance accrue aux différentes formes d'assistance sociale ou de paiements de transfert qui se substituent maintenant aux revenus d'appoint en forêt.

Bref, toutes les industries primaires du Québec ont certains traits communs : l'évolution technologique y est rapide, la production aug mente mais l'emploi diminue. De sérieux problèmes de déplacement et d'adaptation de la main-d'œuvre sont à prévoir dans les années à venir.

Depuis 1950, il est devenu de plus en plus évident que le progrès économique du Québec dépend, dans une large mesure, du développement d'industries manufacturières. La croissance du secteur secondaire permet la création de nouveaux emplois par l'introduc tion de nouveaux procédés de fabrication et de nouveaux produits. Toutefois, cette croissance a ses exigences. L'utilisation de machines de plus en plus complexes, la mécanisation ou l'automatisation demandent une main-d'œuvre spécialisée qui n'est pas toujours prête à assumer de nouvelles fonctions. La main'd'œuvre du secteur primaire, qui se dirige vers le secteur secondaire, doit faire face à des problèmes d'adaptation très sérieux. En outre, les besoins de maind'œuvre par unité de production ont tendance à diminuer dans les industries manufacturières. Le développement de l'emploi n'est done pas aussi rapide qu'on l'aurait espéré. Par exemple, la valeur de la production dans les industries de transformation du Québec a aug. menté de 100 pour cent de 1950 à 1963 : de 4.1 à 8.4 milliards de dollars. Durant la même période, l'emploi s'est accru d'un peu moins de 17 pour cent, ce qui est très peu relativement aux fortes augmentations de la production. L'énergie mécanique se substitue au travail de l'homme. Cette hypothèse peut d'ailleurs se vérifier facilement en étudiant l'évolution des investissements privés et publics dans les industries de fabrication. De 1950 à 1964, le taux 
d'accroissement annuel moyen des immobilisations dans les machines et l'outillage a été d'environ 15 pour cent, alors que celui de l'emploi n'atteint pas 2 pour cent. D'autre part, cette évolution globale du secteur manufacturier cache les faiblesses de certaines industries de transformation. Certaines industries ont plutôt subi une diminution de l'emploi durant cette période (l'industrie du textile), d'autres n'ont fait aucun gain (les industries du tabac, du papier, des métaux non ferreux et des produits chimiques). Il va de soi que les régions qui possèdent ces types d'industries peuvent avoir une croissance économique moins rapide que celles qui ont des industries plus dynamiques et plus diversifiées.

Au total, une diminution de l'emploi au secteur primaire et une faible augmentation au secteur secondaire furent heureusement accompagnées par une très forte augmentation de l'embauche dans les industries du secteur tertiaire ou des services (transports, services publics, commerce, finance, etc.).

De 1951 à 1961, la main-d'œuvre du secteur tertiaire s'est en effet accrue de plus de 40 pour cent. 52 pour cent de la maind'œuvre totale du Québec œuvrent maintenant dans les industries des services. Par comparaison, environ 33 pour cent et 11 pour cent font respectivement partie des secteurs secondaire et primaire. Le tableau I permet de mesurer l'évolution de 1951 à 1961 .

Durant cette période, deux tendances se sont donc produites. Premièrement, l'augmentation nette de l'emploi dans les industries

\section{Tableau I}

Main-d'œuvre par secteur au Québec

\begin{tabular}{l|r|r|r|r}
\hline \hline & \multicolumn{2}{|c|}{1951} & \multicolumn{2}{c}{1961.} \\
\cline { 2 - 5 } & nombre & p.c. & nombre & p.c. \\
\cline { 2 - 5 } & 262,908 & 17.86 & 202,521 & 11.45 \\
Primaire ...... & 526,918 & 35.79 & 592,804 & 33.53 \\
Secondaire ...... & 652,481 & 44.33 & 921,527 & 52.12 \\
Tertiaire ....... & 29,533 & 2.02 & 51,267 & 2.00 \\
Indéfinie ....... & & 100 & $1,763,119$ & 100 \\
\hline Total .......... & $1,471,840$ & 100 & & \\
\hline
\end{tabular}


productrices de biens et dans la construction (les secteurs primaire et secondaire) n'a été que de 5,499 , par opposition à une augmentation de près de 270,000 dans les industries des services. Deuxiè, mement, un fort déplacement de la main d'cuvre a eu lieu à l'intérieur des secteurs primaire et secondaire. Le premier a subi une perte nette de 60,387 emplois, l'autre a bénéficié d'un gain de 65,886 emplois. Théoriquement, l'expansion des industries du secteur secondaire aurait été suffisante pour absorber la main-d'œuvre déplacée du secteur primaire. En pratique, toutefois, il est probable que le secteur secondaire n'a pu recevoir toute cette main-d'cuvre et ce, surtout, par manque de connaissances technologiques d'une forte proportion de cette main-d'œuvre. C'est plutôt la jeune main-d'œuvre spécialisée qui entre dans les industries de transformation. En conséquence, très peu de possibilités s'offrent aux travailleurs déplacés du secteur primaire, sinon le chômage.

$\mathrm{Au}$ Québec, les emplois qui exigent le moins de formation sco laire sont en voie de diminution. Les industries demandent des spécialistes et des travailleurs qualifiés. Et, sous ce rapport, le Québec est défavorisé par rapport au reste du Canada, comme on peut le constater au tableau II.

En 1961, environ 52 pour cent de notre main-d'œuvre n'avaient pas dépassé le seuil du niveau primaire, 28 pour cent avaient complété le secondaire et 8.87 pour cent étaient des universitaires. Au Canada, ces pourcentages étaient respectivement d'environ 44, 46 et 9. Nous sommes donc en retard en comparaison du Canada et l'effort à accomplir dans le domaine éducatif est immense. De cet effort dépend aussi la croissance économique du Québec.

Tableau II

Instruction de la main-d'œuvre, 1961

\begin{tabular}{|c|c|c|c|c|}
\hline & & $\begin{array}{l}\text { Niveau } \\
\text { primaire }\end{array}$ & $\begin{array}{c}\text { Niveau } \\
\text { secondaire }\end{array}$ & $\begin{array}{c}\text { Niveau } \\
\text { universitaire }\end{array}$ \\
\hline Québec $\ldots \ldots \ldots \ldots$ & $\cdot$ & $\begin{array}{l}\text { (p.c.) } \\
52.26 \\
44.25\end{array}$ & $\begin{array}{l}\text { (p.c.) } \\
28.69 \\
46.32\end{array}$ & $\begin{array}{l}\quad \text { (p.c.) } \\
\quad: 8.87 \\
9.29\end{array}$ \\
\hline
\end{tabular}


Pour en revenir au secteur tertiaire, voyons enfin son évolution sur les plans des investissements et de la composition de la maind'œuvre. De 1950 à 1964, il s'en dégage les faits suivants :

- les investissements annuels totaux ont quadruplé ou presque. Ils atteignent maintenant les 835 millions;

- les investissements dans les services d'utilité publique ont été plus rapides que dans les entreprises du commerce de la finance et des services commerciaux ;

- le taux d'accroissement annuel moyen des investissements a été d'environ 20 pour cent durant cette période;

- de la main-d'œuvre travaillant dans le secteur tertiaire, l'aug' mentation la plus sensible eut lieu chez les professionnels et techniciens (58 pour cent), suivis des employés de bureau (46), des travailleurs des services (45), des vendeurs (36), des administrateurs (25) et des travailleurs des transports (20).

Les conclusions qui se dégagent de cette analyse sont que le secteur tertiaire se développe plus rapidement que les deux autres secteurs et que, grâce à ce développement, il est devenu la principale source d'emplois pour notre main-d'œuvre. Si nous voulons éviter de graves problèmes économiques et sociaux, il faut espérer que le rythme de la création d'emplois dans ce secteur se maintienne dans les années à venir.

Un des objectifs les plus pressants de la politique du Québec est le développement de ses diverses régions économiques. Car un degré de participation équilibrée des dix régions économiques à la croissance économique du Québec demeure encore un idéal extrế mement lointain chez nous. En fait, le déséquilibre inter-régional est profond et il a probablement tendance à s'accentuer d'une année à l'autre.

Traditionnellement, le Québec est divisé en deux parties : d'une part, les régions dites concentriques, Montréal et Montréal métropolitain ; d'autre part, les régions dites excentriques, les Cantons'del'Est, Trois-Rivières, Québec, Gaspésie-Rive sud, Saguenay-Lac-St- 


\section{EVOLUTION ECONOMIQUE DU QUÉBEC}

Jean, l'Outaouais, Abitibi-Témiscamingue 'et Côte-Nord-NouveauQuébec. Les régions concentriques sont caractérisées par une croissance économique rapide. Les régions excentriques donnent lieu, sauf de rares exceptions, à un développement économique lent ou même à une certaine stagnation. Ces dernières sont souvent appelées régions sous développées ou défavorisées, impliquant ainsi un revenu per capita relativement bas. Cette mesure statistique du degré de développement économique d'une région, malgré les difficultés d'ordre conceptuel qu'elle présente, est à peu près la seule que nous pouvons utiliser avec une certaine facilité. Selon cette mesure, le revenu personnel brut per capita atteignait environ 1,341 dollars au Québec en 1961. Toutefois, le revenu personnel brut per capita est inférieur à ce montant dans toutes les régions économiques du Québec sauf deux, le Montréal métropolitain et la Côte-Nord-Nouveau-Québec.

Si l'ordre des régions du point de vue du revenu par habitant était maintenant utilisé comme mesure de leur développement, pas sant de la plus défavorisée à la moins défavorisée, on aurait l'ordre suivant : 1) Gaspésie-Rive sud, 2) Abitibi-Témiscamingue, 3) Can-

\section{Tableau III}

Revenu personnel par habitant, selon les régions, $1961^{2}$

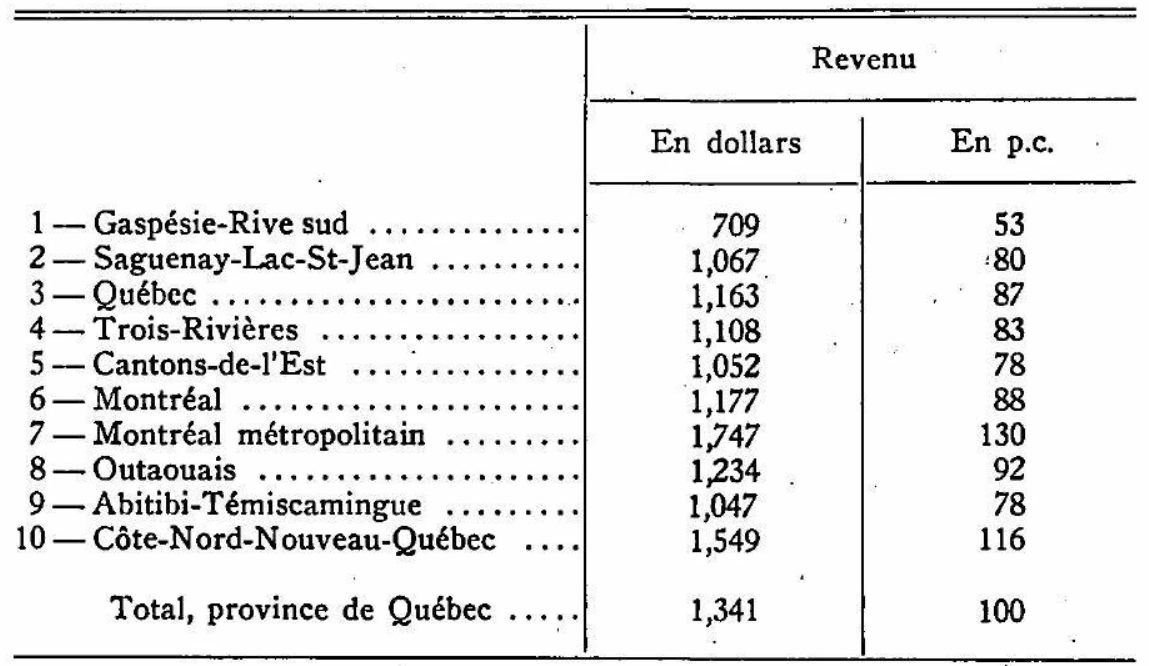

2. Source: Bureau des Recherches Economiques, ministère de l'Industrie et du Commerce, Québec. 
tons-de-l'Est, 4) Saguenay-Lac-St-Jean, 5) Trois-Rivières, 6) Qué bec, 7) Montréal, -8) Outaouais, 9). Côte-Nord-Nouveau-Québec, 10) Montréal métropolitain. Cependant, une part de plus en plus large en paiements de transfert des gouvernements provincial et fédéral tend à masquer la faiblesse relative de l'activité génératrice de revenu dans certaines régions. En somme, le revenu per capita serait beaucoup plus faible qu'il ne l'est présentement si ce n'était l'apport substantiel de l'Etat dans les régions défavorisées. En Gas pésie, par exemple, 30 pour cent du revenu du père de famille provient des paiements de transfert ${ }^{3}$ alors que ce pourcentage n'est que de 10 dans les familles ouvrières québécoises ${ }^{4}$. L'Etat intervient donc pour atténuer, dans une certaine mesure, l'inégalité de revenus des régions du Québec.

Comment expliquer cette inégalité inter-régionale des revenus? Pourquoi certaines régions sont-elles plus pauvres que d'autres? Quels sont les facteurs socióéconomiques qui peuvent expliquer un tel état de choses?

Nos régions excentriques sont affectées par un chômage chro nique. L'accroissement naturel de la population est généralement très élevé ou l'offre de travail s'accroît à un rythme rapide, alors que la demande de travail diminue dans la plupart des secteurs de l'acti' vité économique. Les principales ressources de ces régions, l'agriculture, la forêt, les pêches et les mines ne peuvent absorber les surplus de main-d'ouvre et perdent même chaque année une partie de leurs effectifs. Les industries primaires utilisent relativement moins de main-d'œuvre. Le secteur secondaire est faible ou fortement mé canisé. Les industries saisonnières dominent aussi tout le processus économique régional. Il existe donc un déséquilibre profond entre l'offre et la demande de travail. La preuve en est faite lorsque l'on examine la situation de l'emploi et du chômage en Gaspésie, par exemple. Les chefs de famille travaillent en moyenne 29 semaines et en chôment 23 , soit près de six mois de chômage ${ }^{5}$. De ce fait, la main-d'œuvre de nos régions défavorisées doit de toute nécessité

3. Daneau, Marcel, Situation économique des familles rurales de la Gaspésie, 1963 (inédit).

4. Tremblay, M.A. et Fortin, Gérald, Les comportements économiques de la famille salariée du 2uébec, Presses de l'Université Laval, I965. (inédit).

5. Daneau, Marcel, Situation économique des familles rurales de la Gaspésie, 1963 
se diriger vers les régions industrielles, soit Montréal ou le Montréal métropolitain, pour se trouver du travail. Mais ce mouvement de population ne fut pas assez rapide pour éliminer le chômage, déguisé ou non, qui sévit dans les régions excentriques. Et cette importante main-d'œuvre résiduelle pose des problèmes extrêmement sérieux d'utilisation à l'intérieur des territoires concernés. Néanmoins, l'em. ploi de cette main d'œuvre serait possible par le réaménagement des ressources existantes, le développement d'industries de transformation de ces mêmes ressources et par l'implantation d'autres indus. tries non axées spécifiquement sur l'utilisation des matières premières de ces territoires, ou par la décentralisation industrielle. Dans la conjoncture économique actuelle, il semble que la décentralisation industrielle pose le plus grand nombre de difficultés. Il est vrai que certaines politiques fiscales, tels le moratoire fiscal, la dépréciation accélérée et les subsides directs de l'État sont d'excellentes mesures de décentralisation mais, par elles-mêmes, il est douteux qu'elles puissent agir efficacement. Au Québec, l'attrait du marché et la rentabilité des entreprises demeurent les facteurs les plus importants de localisation de l'entreprise privée. Sous ce rapport, il est possible que la décentralisation repose surtout sur l'entreprise étatique comme moyen d'alléger le manque d'emplois des régions sousdéveloppées du Québec. Bref, la faiblesse des revenus per capita de la plupart des régions économiques du Québec provient d'un manque de capital, d'une population excédentaire par rapport aux ressources disponibles, d'absence de ressources adéquates et de progrès technologique rapide. Cependant, ces facteurs agissent différemment selon les régions et expliquent les variations inter-régionales de la croissance économique. Malheureusement, la comptabilité régionale n'existe pas pour corroborer ces faits. Les seuls outils statistiques disponibles sont l'emploi et la valeur de la production dans les industries de transformation. Ces chiffres donnent quand même une idée de l'évolution industrielle des diverses régions économiques du Québec.

Nous avons examiné précédemment la régression de l'emploi dans l'agriculture et l'exploitation forestière. Il va de soi que les régions dominées par ces industries ont des problèmes d'ajustements très sérieux. $\mathrm{Si}$, en même temps, ces régions doivent aussi subir une diminution de l'emploi dans le secteur des industries manufacturiè- 
res, les problèmes deviennent doublement sérieux. Dans les cas de la Gaspésie-Rive sud, de l'Outaouais, de l'Abitibi-Témiscamingue, l'emploi a diminué d'environ 9 pour cent dans les industries de transformation de 1950 à 1960. Dans les régions du Saguenay-Lac' St-Jean, des Trois-Rivières et des Cantons-de-l'Est, l'emploi aug' mente très peu ou a eu tendance à se stabiliser au cours des dernières années. Finalement, les régions de Montréal, de Montréal métropolitain, de Québec et de la Côte-Nord-Nouveau-Québec semblent jouir d'une expansion de l'emploi relativement plus forte que les autres régions dans le secteur des industries de transformation. En résumé, il est évident que la croissance économique a des intensités différentes selon les régions. Il apparaît aussi que l'axe Montréal-Québec prend de plus en plus la forme d'un immense pôle de développement et d'attraction pour la main-d'œuvre de tout le Québec. Ce processus dynamique de concentration a influencé l'évo lution économique du Québec pendant les quinze dernières années. Il est d'ores et déjà assuré que ce processus la conditionnera encore durant de très nombreuses années.

En terminant, notons les principaux facteurs qui expliquent le niveau des revenus per capita de chacune des dix régions économiques du Québec.

La région de la Gaspésie-Rive sud a une économie axée sur les industries du secteur primaire : agriculture, pêche, forêt et mines. L'agriculture y est généralement pauvre ou marginale, sauf sur une mince lisière qui borde le fleuve $\mathrm{St}$-Laurent et la baie des Chaleurs. Le domaine forestier est exploité à pleine capacité et montre même des signes graves de surexploitation. L'industrie de la pêche a pris une certaine expansion durant ces dernières années mais elle atteint présentement le point de saturation. Le développement du secteur minier a eu peu d'effet sur l'économie régionale. Le secteur secondaire est faible mais le secteur tertiaire s'est assez bien développé dans certaines villes de services telles Rimouski, Rivière-du-Loup et Gaspé. Il y a émigration des éléments jeunes et actifs de la population.

$\mathrm{Au}$ Saguenay-Lac-St-Jean, l'agriculture, la forêt et les mines jouent un rôle de premier plan dans l'activité économique. L'agriculture est surtout prospère dans la plaine alluviale du Lac-St-Jean puis elle dépérit graduellement sur les flancs rocheux des Lauren. 
tides. Les fermes marginales qui s'y trouvent retirent leurs revenus en grande partie de l'exploitation forestière. Le secteur secondaire est peu diversifié, se limitant à deux types de production ; la fabrication de l'aluminium et des pâtes et papiers. Ce sont des industries utilisatrices de capital et non de main-d'cuvre. L'absence de diversité industrielle fait que le ralentissement de la production dans l'une ou l'autre de ces industries a un effet très considérable sur toute l'économie régionale.

Dans la région de Québec on a affaire encore une fois à un milieu agricole et rural, relativement prospère en bordure du St-Lau rent et pauvre dans l'arrière'pays. La ville de Québec y joue le rôle de pôle majeur d'attraction et d'influence des activités économiques et de la main-d'œuvre. L'industrie des services s'y est, par conséquent, bien développée sans qu'il n'y ait de véritable contrepartie dans le secteur industriel. La région de Québec ne manifeste donc pas tout le dynamisme qu'on pourrait espérer.

Les villes des Trois-Rivières et de Shawinigan fournissent à la région de la Mauricie une prospérité enviable grâce à un certain nombre d'entreprises plus ou moins diversifiées comme les pâtes et papiers, les textiles et les produits chimiques. $\grave{A}$ faible distance de Montréal et localisée autour de centres urbains moyens, l'agriculture y trouve un débouché pour ses produits et les revenus des cultivateurs sont plus élevés que dans les régions de Québec, du Saguenay. Lac-St-Jean et autres régions périphériques. L'exploitation forestière est aussi plus rentable qu'ailleurs à cause de la proximité des usines transformatrices du bois. De ce fait, les revenus per capita sont à peu près de même niveau que ceux de la région de Montréal.

Les Cantons-de-l'Est, région montagneuse, vivent d'une agriculture moins prospère que celle des régions de Montréal, des Trois-Rivières et de Québec dans l'ensemble. On y trouve un certain nombre de villes moyennes, Sherbrooke, Drummondville et Granby, qui réunissent des entreprises anciennes (industrie des textiles) et nouvelles (industries légères). Les centres d'industries légères paraissent plus dynamiques que les autres. Le manque d'industries motrices et lourdes est un handicap sérieux au développement économique de la région.

La région de Montréal bénéficie de la concentration et du débordement des activités de la ville de Montréal. La formation de 


\section{L'ACTUALITE ECONOMIQUE}

villes satellites autour du grand centre en est une excellente preuve. L'agriculture de la région de Montréal est prospère dans l'énsemble. Les riches basses terres alluviales du St-Laurent, le climat, les marchés lui fournissent les éléments essentiels de sa richesse. Le secteur primaire, toutefois, est nettement relégué au troisième rang après le secondaire et le tertiaire.

Montréal est l'agglomération la plus importante, la plus diver. sifiée et la plus dynamique du Québec. Elle possède les facteurs traditionnels de localisation, la main-d'œuvre, les marchés, les capitaux, le personnel technique et administratif, enfin tous ces critères qui en font le centre polarisateur des activités économiques de la province. La concentration industrielle commerciale et financière a fait de Montréal, à toute fin pratique, le grand centre de décision du Québec.

Les revenus per capita de la région de l'Outaouais sont passablement élevés, grâce à l'apport de la ville d'Ottawa dans le comté environnant de Hull. Les fonctionnaires canadiens-français y ont établi leur résidence de même que certains services fédéraux. D'autre part, l'agriculture n'est guère rentable dans les comtés de Gatineau et de Pontiac et le revenu par acre est même inférieur à celui de la Gaspésie-Rive sud. L'exploitation forestière occupe encore une place dominante dans l'économie régionale.

Région minière, agricole et forestière, peu industrialisée, l'Abitibi-Témiscamingue est aussi victime de l'éloignement des principaux centres de développement: Cet éloignement freine sa croissance éco nomique. Son économie est centrée sur l'exploitation des matières premières dont la valeur ajoutée est moindre que sur les produits transformés. En conséquence, les revenus per capita sont inférieurs à la moyenne provinciale et rejoignent à peine le niveau atteint dans la région du Saguenay-Lac-St-Jean.

L'industrie minière, enfin, a bouleversé l'économie de la CôteNord-Nouveau-Québec. Les salaires versés par cette industrie sont élevés et attirent une main d'œuvre nombreuse des régions adjacentes. La population s'est accrue considérablement et des villes comme Baie-Comeau, Sept-illes, Gagnonville sont devenues de véritables petits pôles de croissance et d'attraction. Leurs effets de polarisation sont cependant limités par l'absence d'industries de trans' formation du minerai. 


\title{
EVOLUTION ECONOMIQUE DU 'QUEBEC
}

Cette rapide comparaison des diverses régions économiques du Québec nous révèle, en résumé, qu'il y a un déséquilibre profond entre la zone métropolitaine de Montréal et les régions périphériques. Ce déséquilibre résulte d'un ensemble de facteurs socióéconomiques qui font bénéficier la ville de Montréal d'une croissance rapide et les autres d'une expansion lente. Le déséquilibre intra-régio nal est aussi très grave. $A$ l'intérieur des régions économiques, certaines villes s'appauvrissent, d'autres se développent. Il nous apparaît urgent d'appuyer le développement des: villes dynamiques qui peuvent devenir de véritables pôles de croissance régionale.

\author{
Marcel DANEAU, \\ professeur à l'Université Laval
}

\author{
APPENDICE \\ (voir pages 682 à 692)
}


L'ACTUALITE ECONOMIQUE

\begin{tabular}{|c|c|c|c|c|c|c|c|c|c|c|}
\hline & \% & 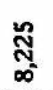 & 蒿 & 疋 & $\stackrel{\hat{\circ}}{\circ}$ & 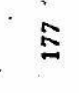 & in & $\approx$ & $\stackrel{0}{0}$ & \\
\hline & ๙ั้ & $\begin{array}{l}9 \\
\frac{9}{2} \\
\end{array}$ & $\underset{\vdots}{\vdots}$ & $\stackrel{0}{\vdots}$ & $\begin{array}{l}\infty \\
\text { n. } \\
\text { n. }\end{array}$ & $\bar{\Xi}$ & ff & $\bar{\Omega}$ & : & \\
\hline & $\bar{\circ}$ & 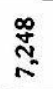 & $\stackrel{\substack{m \\
:}}{-\infty}$ & : & & $\stackrel{\Phi}{\Phi}$ & $\overline{\mathcal{N}}$ & $\bar{n}$ & gे & \\
\hline & ळ. & 兽 & $\stackrel{9}{9}$ & 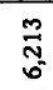 & \begin{tabular}{l}
$\infty$ \\
$\stackrel{\infty}{*}$ \\
\multirow{\sigma}{*}{}
\end{tabular} & $\stackrel{\infty}{\stackrel{2}{2}}$ & ঞ̊ & $\mathscr{\mathscr { O }}$ & $\stackrel{2}{2}$ & \\
\hline & ํำ & 您 & 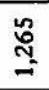 & जू & fa & $\underset{\sim}{\widetilde{\Phi}}$ & 8 & $\frac{\sim}{0}$ & $\overline{\boldsymbol{\Phi}}$ & \\
\hline & $\stackrel{\infty}{\stackrel{\infty}{\Omega}}$ & $\begin{array}{l}\overrightarrow{0} \\
0 \\
0\end{array}$ & $\underset{\substack{\infty \\
-}}{-\infty}$ & $\begin{array}{l}\hat{0} \\
\text { if }\end{array}$ & ্ָণু & $\stackrel{-}{\prime}$ & 号 & 品 & ฉి & \\
\hline ڤ్ర & 总 & $\begin{array}{l}\mathcal{y} \\
\text { is }\end{array}$ & 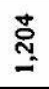 & $\begin{array}{l}\vec{w} \\
\text { s. } \\
\text { s. }\end{array}$ & $\frac{\pi}{\sigma}$ & 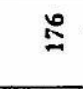 & $\vec{b}$ & $\frac{ \pm}{n}$ & 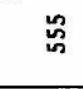 & \\
\hline & 号 & in & $\stackrel{g}{=}$ & @ั & $\underset{\substack{0 \\
\infty \\
\infty}}{0}$ & $\stackrel{Ð}{\varpi}$ & \%ి & 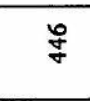 & $\underset{\mathscr{\alpha}}{\mathscr{\alpha}}$ & \\
\hline \&్ & 蒿 & \begin{tabular}{l}
$\hat{\jmath}$ \\
\multirow{\sigma}{*}{} \\
$\forall$
\end{tabular} & :్- & $\frac{8}{8}$ & 柋 & $\stackrel{\infty}{\sim}$ & $\stackrel{\text { ॥ू }}{\circ}$ & 啇 & б్ & \\
\hline • & 获 & 捈 & ò. & $\vec{m}$ & $\underset{N}{m}$ & $\overline{\mathrm{N}}$ & 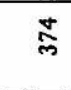 & $\frac{9}{q}$ & $\frac{m}{7}$ & $\widetilde{\sigma}$ \\
\hline & 总 & ơ & $\stackrel{0}{*}$ & $\stackrel{\circ}{\circ}$ & $\underset{N}{N}$ & $\tilde{\widetilde{N}}$ & 离 & ষ্ণ & ఏ్లి & \\
\hline 总总 & ్ㅐㅇ & $\stackrel{\pi}{\frac{N}{5}}$ & ณू & $\vec{D}$ & 商 & 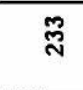 & 悉 & 足 & స్ల & \\
\hline & $\overrightarrow{\mathrm{g}}$ & 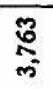 & 沉 & 岕 & 芯 & 莡 & ম్ల & ᄋ్ల & $\underset{\mathrm{N}}{\mathbf{t}}$ & \\
\hline 昜 & $\begin{array}{l}\text { 足 } \\
\text { 品 }\end{array}$ & $\stackrel{\vec{m}}{m}$ & ○ & $\stackrel{\substack{n \\
m}}{n}$ & ָָ & \%ั้ & 听 & 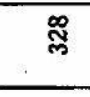 & 岕 & \\
\hline 8 & & 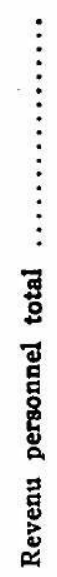 & 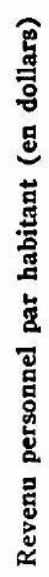 & 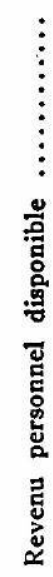 & 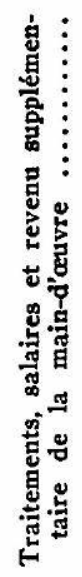 & 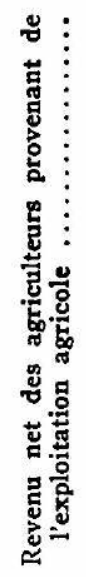 & 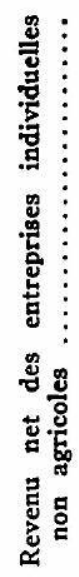 & 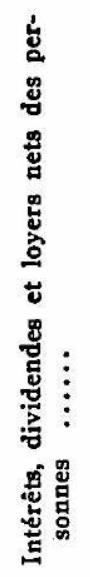 & 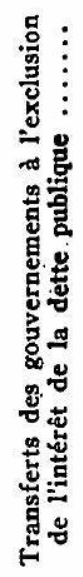 & 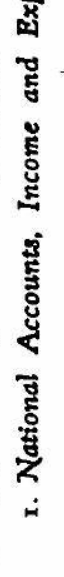 \\
\hline
\end{tabular}


EVOLUTION ECONOMIQUE DU QUEBEC

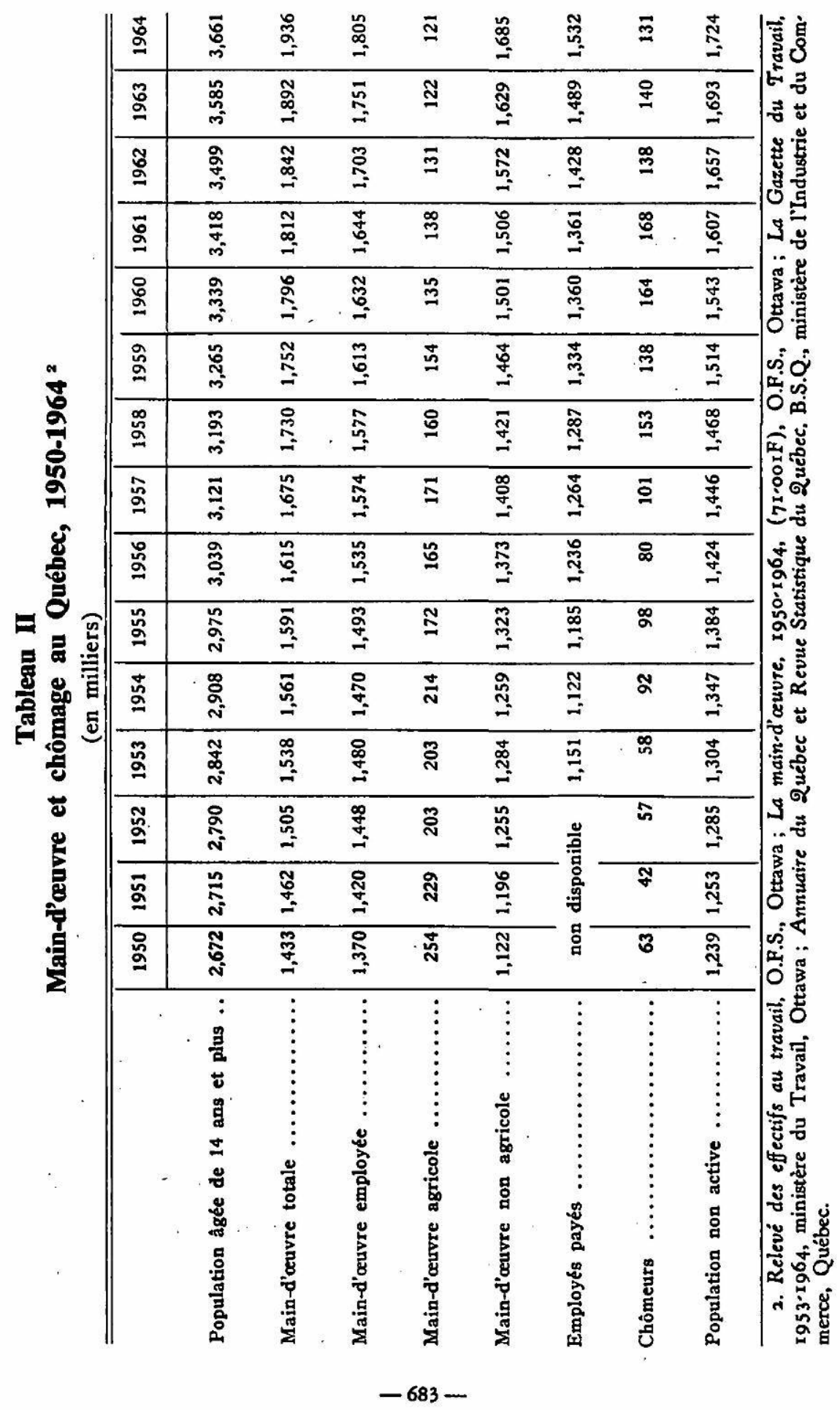




\section{LACTUALITE ECONOMIQUE $1 \cdots$}

\section{Tableau III}

\section{Les finances publiques au Québec, 1950-1963}

(en milliers de dollars)

\begin{tabular}{|c|c|c|c|c|c|}
\hline . & $\begin{array}{c}\text { Dépenses } \\
\text { totales } \\
\text { municipales }\end{array}$ & $\begin{array}{c}\text { Dépenses tota- } \\
\text { les générales } \\
\text { brutes provin- } \\
\text { ciales }\end{array}$ & & $\begin{array}{c}\text { Dépenses } \\
\text { totales } \\
\text { municipales }\end{array}$ & $\begin{array}{l}\text { Dépenses tota- } \\
\text { les générales } \\
\text { brutes provin- } \\
\text { ciales }\end{array}$ \\
\hline 1950 & - & 270,597 & 1957 & 319,311 & 539,987 \\
\hline 1951 & 184,951 & 303,452 & 1958 & 352,799 & 587,245 \\
\hline 1952 & 202,248 & 342,598 & 1959 & 415,298 & 669,321 \\
\hline 1953 & 214,626 & 345,011 & 1960 & 434,209 & 832,077 \\
\hline 1954 & 246,771 & 384,957 & 1961 & 467,063 & $1,028,458$ \\
\hline 1955 & 261,854 & 439,655 & 1962 & 466,885 & 二- \\
\hline .1956 & 290,430 & 475,474 & 1963 & 496,406 & - \\
\hline
\end{tabular}

Tableau IV

Investissements privés et publics au Québec, 1950-1964, dépenses de réparation et d'entretien exclues "

(en millions de dollars)

\begin{tabular}{l|c|c|c||c|c|c|c}
\hline \hline & $\begin{array}{c}\text { Cons- } \\
\text { truction }\end{array}$ & $\begin{array}{c}\text { Machine- } \\
\text { rie et } \\
\text { équipe- } \\
\text { ment }\end{array}$ & Total & & $\begin{array}{c}\text { Cons- } \\
\text { truction }\end{array}$ & $\begin{array}{c}\text { Machine- } \\
\text { rie et } \\
\text { équipe- } \\
\text { ment }\end{array}$ & $\begin{array}{c}\vdots \\
\text { Total }\end{array}$ \\
\cline { 2 - 8 } 1950 & 558 & 288 & 846 & 1958 & 1,437 & $\ddots 617$ & 2,054 \\
1951 & 692 & 373 & 1,065 & 1959 & 1,460 & 634 & 2,094 \\
1952 & 843 & 440 & 1,283 & 1960 & 1,327 & 680 & 2,007 \\
1953 & 891 & 483 & 1,374 & 1961 & 1,386 & 622 & 2,008 \\
1954 & 938 & 424 & 1,362 & 1962 & 1,512 & 642 & 2,154 \\
1955 & 1,074 & 472 & 1,546 & $1963^{\circ}$ & 1,569 & 712 & 2,281 \\
1956 & 1,260 & 591 & 1,851 & $1964^{\circ}$ & 1,904 & 782, & 2,686 \\
1957 & 1,377 & 652 & 2,029 & & & & \\
\hline
\end{tabular}

3. Financial Statistics of Municipal Governments, $195 \times 1963,(68 \cdot 203)$, O.F.S., Ottawa.

4. Financial Statistics of Provincial Governments, r950-196r (68.207), O.P.S., Ottawa.

5. Investissements privés et publics au Canada, 1950.1964; ministère du Com. merce, Ottawa.

6. Chiffres préliminaires.

7. Prévisions. 
EVOLUTION ECONOMIQUE DU QUÉBEC

\section{Tableau V \\ Epargne au Québec, 1949-1962:}

(en millions de dollars)

\begin{tabular}{l|c|c||c|c|c}
\hline \hline & $\begin{array}{c}\text { Epargne } \\
\text { nette des } \\
\text { personnes }\end{array}$ & $\begin{array}{c}\text { Epargne } \\
\text { des } \\
\text { entreprises }\end{array}$ & & $\begin{array}{c}\text { Epargne } \\
\text { nette des } \\
\text { personnes }\end{array}$ & $\begin{array}{c}\text { Epargne } \\
\text { des } \\
\text { entreprises }\end{array}$ \\
\cline { 2 - 5 } 1949 & 224.9 & 459.41 & 1958 & - & 701.0 \\
1951 & 318.8 & 531.74 & 1959 & 430.9 & 758.1 \\
1953 & 322.8 & 644.96 & 1960 & -7.9 & 704.7 \\
1955 & 215.1 & - & 1961 & 437.9 & 719.1 \\
1956 & - & 682.2 & 1962 & 425.0 & - \\
1957 & 298.4 & 699.3 & & & \\
\hline
\end{tabular}

\section{Tableau VI}

Les dépenses de consommation au Québec, 1950-1963

(en milliers de dollars)

\begin{tabular}{l|c|c||c|c|c}
\hline \hline & $\begin{array}{c}\text { Ventes au détail effectuées } \\
\text { au Québec }\end{array}$ & & \multicolumn{2}{|c|}{$\begin{array}{c}\text { Ventes au détail effectuées } \\
\text { au Québec }\end{array}$} \\
\cline { 2 - 6 } & $\begin{array}{c}\text { Ventes } \\
\text { totales }\end{array}$ & $\begin{array}{c}\text { Véhicules } \\
\text { automobiles } \\
\text { neufs }{ }^{10}\end{array}$ & & $\begin{array}{c}\text { Ventes } \\
\text { totales }\end{array}$ & $\begin{array}{c}\text { Véhicules } \\
\text { automobiles } \\
\text { neufs }{ }^{\text {to }}\end{array}$ \\
\cline { 2 - 6 } 1950 & $2,182,983$ & 166,992 & 1957 & $3,521,412$ & 359,479 \\
1951 & $2,442,649$ & 189,770 & 1958 & $3,646,651$ & 328,237 \\
1952 & $2,335,302$ & 207,196 & 1959 & $3,877,558$ & 344,489 \\
1953 & $2,756,136$ & 228,224 & 1960 & $3,944,345$ & 376,597 \\
1954 & $2,797,617$ & 214,362 & 1961 & $4,108,857$ & 399,428 \\
1955 & $3,005,671$ & 272,505 & 1962 & $4,444,800$ & 466,421 \\
1956 & $3,222,150$ & 346,745 & 1963 & $4,772,500$ & 526,848 \\
\hline
\end{tabular}

8. 2uébec, La situation économique, 196r, 1962, Bureau de Recherches économiques, ministère de l'Industrie et du Commerce, Québec.

9. Retail Trade, 195011963, (63.005), O.F.S., Ottawa.

ro. New Motor Vehicle Sales, 1950-1963, $(63 \cdot 208)$, O.F.S., Ottawa.

(Notes des tableaux VII et VIII, p. 686)

rx. Farm Net income, 1950-1963, (21-302), O.P.S., Ottawa.

12. Index of Farm Production, 1950-1963, (21/203), O.F.S., Ottawa.

13. Index Numbers of Farm Prices of Agricultural Products, 1950.1964, (62.003), O.F.S., Ottawa.

14. La main-d'cuvre, $1950 \cdot 1963$, (71-00 rF), O.P.S., Ottawa.

15. Pêcheries maritimes, 1958.1960, et Pếcheries commerciales, 196r et 1962, B.S.Q., ministère de I'Industrie et du Commerce, Québec; Québec, La situation éco. nomique, 1963 . Bureau de Recherches économiques, idem.

16. Pêcheries maritimes, 1958-1960, B.S.Q., ministère de l'Industrie et du Commerce, Québec.

17. Daneau, Marcel, \&L'industrie des pêches maritimes du Québec \$, L'Actualité Economique, janvier-mars r96r. 
L'ACTUALITÉ ÉCONOMIQUE.

Tableau VII

L'agriculture, Québec, 1950-1964

\begin{tabular}{l|c|c|c|c|c}
\hline & \multicolumn{2}{|c|}{$\begin{array}{c}\text { Revenu total } \\
\text { (en milliers de dollars) }\end{array}$} & $\begin{array}{c}\text { Indice de la } \\
\text { production }\end{array}$ & $\begin{array}{c}\text { Indice des } \\
\text { prix a la } \\
\text { ferme }\end{array}$ & $\begin{array}{c}\text { Main- } \\
\text { d'cuvre }\end{array}$ \\
\cline { 2 - 3 } & Brut & Net & (1949=100) & $(1935-39=100)$ & (en milliers) \\
\cline { 2 - 5 } 1950 & 403,350 & 211,416 & 108.0 & 260.9 & 254 \\
1951 & 482,186 & 261,476 & 110.6 & 305.6 & 229 \\
1952 & 462,362 & 241,888 & 116.2 & 290.2 & 203 \\
1953 & 447,780 & 238,144 & 113.3 & 272.1 & 203 \\
1954 & 445,188 & 216,442 & 110.9 & 264.3 & 214 \\
1955 & 462,249 & 228,482 & 124.6 & 261.7 & 172 \\
1956 & 470,866 & 193,630 & 127.2 & 258.8 & 165 \\
1957 & 457,718 & 185,035 & 128.0 & 264.8 & 171 \\
1958 & 503,912 & 203,699 & 133.8 & 274.8 & 160 \\
1959 & 503,340 & 191,848 & 134.3 & 272.7 & 154 \\
1960 & 499,029 & 185,686 & 134.5 & 273.6 & 135 \\
1961 & 521,169 & 179,073 & 143.3 & 274.4 & 138 \\
1962 & 538,339 & 179,668 & 152.3 & 274.7 & 131 \\
1963 & 554,290 & 189,052 & 154.8 & 274.2 & 122 \\
1964 & - & - & - & 274.2 & 121 \\
\hline
\end{tabular}

\section{Tableau VIII}

Les pêcheries maritimes, Québec, 1950-1963

\begin{tabular}{l|c|c|c|c}
\hline \hline & $\begin{array}{c}\text { Valeur totale } \\
\text { à } \\
\text { 1'expédition's } \\
\text { (en milliers de } \\
\text { dollars) }\end{array}$ & $\begin{array}{c}\text { Immobili- } \\
\text { sations } \\
\text { (en milliers de } \\
\text { dollars) }\end{array}$ & $\begin{array}{c}\text { Indices des prix } \\
\text { moyens payés } \\
\text { aux pêcheurs } \\
(1947-1949=100)\end{array}$ & $\begin{array}{c}\text { Nombre } \\
\text { des } \\
\text { pêcheurs }\end{array}$ \\
\hline 1950 & 4,992 & 3,290 & 86 & 6,810 \\
1951 & 4,968 & 3,248 & 105 & 5,525 \\
1952 & 5,648 & 3,166 & 90 & 4,935 \\
1953 & 5,168 & 3,626 & 93 & 5,053 \\
1954 & 4,615 & 3,590 & 97 & 4,955 \\
1955 & 5,689 & 3,795 & 82 & 4,884 \\
1956 & 7,343 & 4,461 & 99 & 5,290 \\
1957 & 7,125 & 4,664 & 91 & 5,580 \\
1958 & 7,411 & 5,194 & 107 & 6,172 \\
1959 & 7,413 & 5,700 & - & 6,193 \\
1960 & 7,087 & 6,788 & - & 5,387 \\
1961 & 7,649 & - & - & 6,131 \\
1962 & 9,249 & - & - & 6,037 \\
1963 & 10,000 & - & - & 6,500 \\
\hline
\end{tabular}

Notes I I à 17 : voir page 685 . 
EVOLUTION ECONOMIQUE DU QUEBEC

Tableau IX

Les mines, Québec, 1950-1963

\begin{tabular}{c|c|c|c}
\hline \hline & $\begin{array}{c}\text { Valeur totale } \\
\text { annuelle } \\
\text { de la production" } \\
\text { (en milliers de dollars) }\end{array}$ & $\begin{array}{c}\text { Capitaux déboursés } \\
\text { par les prin- } \\
\text { cipaux exploitants" } \\
\text { (en milliers de dollars) }\end{array}$ & $\begin{array}{c}\text { Ouvriers employés } \\
\text { dans les mines } \\
\text { et carrières }\end{array}$ \\
\cline { 2 - 4 } 1950 & 220,665 & - & 26,443 \\
1951 & 255,932 & 31,972 & 28,498 \\
1952 & 270,740 & 48,648 & 30,810 \\
1953 & 252,354 & 48,201 & 29,088 \\
1954 & 278,933 & 69,644 & 29,680 \\
1955 & 357,562 & 61,250 & 31,103 \\
1956 & 423,004 & 59,365 & 31,743 \\
1957 & 406,489 & 102,932 & 34,726 \\
1958 & 366,002 & 61,699 & 31,927 \\
1959 & 441,300 & 71,981 & 32,984 \\
1960 & 446,637 & 84,280 & 32,245 \\
1961 & 455,523 & 64,710 & 31,898 \\
1962 & 519,146 & - & - \\
1963 & 529,388 & - & - \\
\hline
\end{tabular}

Tablean $\mathbf{X}$

La forêt, Québec, 1950-1963

\begin{tabular}{|c|c|c|c|c|c|}
\hline & \multirow{2}{*}{$\begin{array}{c}\text { Valeur totale } \\
\text { de la } \\
\text { productionn } \\
\text { (en milliers de } \\
\text { dollars) }\end{array}$} & \multicolumn{2}{|c|}{ Pâtes et papiers } & \multicolumn{2}{|c|}{ Bois de sciage } \\
\hline & & $\left|\begin{array}{c}\text { Valeur brute } \\
\text { de la } \\
\text { production" } \\
\text { (en milliers de } \\
\text { dollars) }\end{array}\right|$ & $\begin{array}{c}\text { Valeur nette } \\
\text { de la } \\
\text { production } \\
\text { (en milliers de } \\
\text { dollars) }\end{array}$ & $\begin{array}{c}\text { Valeur } \\
\text { de la } \\
\text { production" } \\
\text { (en milliers de } \\
\text { dollars) }\end{array}$ & $\begin{array}{l}\text { Nombre } \\
\text { d'employés" }\end{array}$ \\
\hline 1950 & 509,428 & 421,721 & 221,296 & 65,563 & 19,615 \\
\hline 1951 & 650,195 & 524,164 & 272,958 & 82,374 & 19,358 \\
\hline 1952 & 645,065 & 508,124 & 244,707 & 78,156 & 18,025 \\
\hline 1953 & 635,568 & 511,475 & 251,527 & 82,269 & 17,553 \\
\hline 1954 & 648,434 & 532,327 & 270,538 & 74,988 & 16,529 \\
\hline 1955 & 677,849 & 560,900 & 288,689 & 70,858 & 15,841 \\
\hline 1956 & 733,518 & 622,926 & 308,042 & 78,766 & 13,805 \\
\hline 1957 & 717,986 & 597,119 & 292,452 & 74,960 & 13,351 \\
\hline 1958 & 653,537 & 557,233 & 280,961 & 66,002 & 12,347 \\
\hline 1959 & 868,903 & 585,960 & 294,232 & 72,148 & 12,377 \\
\hline 1960 & 953,994 & 601,188 & 305,845 & 75,103 & 13,089 \\
\hline 1961 & - & 620,710 & 317,192 & 74,291 & 12,584 \\
\hline 1962 & - & - & - & 83,070 & 13,986 \\
\hline 1963 & - & - & - & 88,851 & 12,255 \\
\hline
\end{tabular}

Notes 18 à 24 : voir page 688 . 


\section{Tableau XI}

\section{Production, investissements et emploi, industries manufacturières, Québec, 1950-1963}

\begin{tabular}{|c|c|c|c|c|c|}
\hline & \multirow{2}{*}{$\begin{array}{c}\text { Valeur } \\
\text { de la } \\
\text { production } \\
\text { (en milliers de } \\
\text { dollars) }\end{array}$} & \multicolumn{3}{|c|}{$\begin{array}{l}\text { Investissements privés et publics, dépenses } \\
\text { de réparation et d'entretien exclues }{ }^{=0} \\
\text { (en milliers de dollars) }\end{array}$} & \multirow{2}{*}{$\begin{array}{c}\text { Nombre } \\
\text { d'employés } \\
\text { (cadres admi } \\
\text { nistratifs et } \\
\text { ouvriers a la } \\
\text { production) }\end{array}$} \\
\hline & & $\begin{array}{c}\text { Cons- } \\
\text { truction }\end{array}$ & $\begin{array}{l}\text { Machines et } \\
\text { outillage }\end{array}$ & Total & \\
\hline 1950 & $4,142,473$ & 44.0 & 108.5 & 152.5 & 390,163 \\
\hline 1951 & $4,919,157$ & 54.7 & 143.8 & 198.5 & 417,182 \\
\hline 1952 & $5,176,235$ & 66.0 & 164.9 & 230.9 & 429,698 \\
\hline 1953 & $5,386,787$ & 44.9 & 140.2 & 185.1 & 441,555 \\
\hline 1954 & $5,395,786$ & 72.4 & 131.5 & 203.9 & 424,095 \\
\hline 1955 & $5,922,367$ & 96.3 & 167.8 & 264.1 & 429,575 \\
\hline 1956 & $6,622,503$ & 114.1 & 222.6 & 336.7 & 446,137 \\
\hline 1957 & $6,679,595$ & 119.6 & 256.2 & 375.8 & 449,422 \\
\hline 1958 & $6,754,799$ & 83.4 & 217.2 & 300.6 & 429,358 \\
\hline 1959 & $6,916,196$ & 107.2 & 211.6 & 318.8 & 431,323 \\
\hline 1960 & $7,206,096$ & 86.4 & 218.2 & 304.6 & 433,949 \\
\hline 1961 & $7,349,600$ & 77.2 & 215.1 & 292.3 & 436,124 \\
\hline 1962 & $8,084,500$ & 93.9 & 239.8 & 333.7 & 453,739 \\
\hline 1963 & $8,432,900$ & $82.3^{\mathrm{xs}}$ & $268.4^{23}$ & $350.7^{28}$ & 454,919 \\
\hline 1964 & - & $99.5^{20}$ & $328.8^{\circ}$ & $428.3^{\circ}$ & - \\
\hline
\end{tabular}

25. Répartition et évolution de l'emploi, de la valeur de production, cahier no 4, Bureau de Recherches économiques, ministère de l'Industrie et du Commerce, Québec ; Industries manufacturières du Canada, section C, province de Québec 196r, (31.205), O.F.S., Ottawa; Québec, La situation économique 1963, op. cit.

26. Investissements privés et publics au Canada, r950-1964, ministère du Commerce, Ottawa.

27. Répartition et évolution de l'emploi, de la valeur de production, op. cit.; Québec, La situation économique, 1963. op. cit.

28. Chiffres préliminaires.

29. Prévisions.

(Notes des tableaux IX et X, p. 687)

18. Industrie minière de la province de 2uébec en $196 \mathrm{r}$, ministère dés Richesses naturelles, Québec, 1963 ; Rappơrt préliminaire de la production minérale, 1962, $\left(26.20_{3}\right)$, O.F.S., Ottawa; Québec, La situation économique, rg63, B.R.E., ministère de l'Industrie et du Commerce, Québec.

ig. Industrie minièré de la province de $2 u e ́ b e c$ en $196 \mathrm{r}$, op. cit.

20. L'industrie minière de la province de Québec, 1950-1961, ministère des Richesses naturelles, Québec.

2r. Rapport aninuel du ministre des Terres et Forêts de la province de Québec r $950 \cdot 196 \mathrm{r}$.

22. Annuaire du 2uébec 1963, B.S.2., ministère de l'Industrie et du Commerce, Québec; Les usines de pâtes et papiers, (36.204), O.F.S., Ottawa.

23. Rapport du ministre des Terres et Forêts, r960.196r, Québec: Québec, La situation économique 1963 , op. cit. : Opérations en forêt, (25-201), O.F.S., Ottawa.

24. Rapport du ministre des Terres et Forêts, r960-196r, Québec; Québec, La situation économique $\mathrm{r}^{96} 3$, op. cit. 


\section{EVOLUTION ECONOMIQUE DU QUEBEC}

\section{Tableau XII}

\section{Investissements privés et publics au Québec,}

secteur tertiaire, 1950-1964, dépenses de réparation et d'entretien exclues ${ }^{30}$

(en millions de dollars)

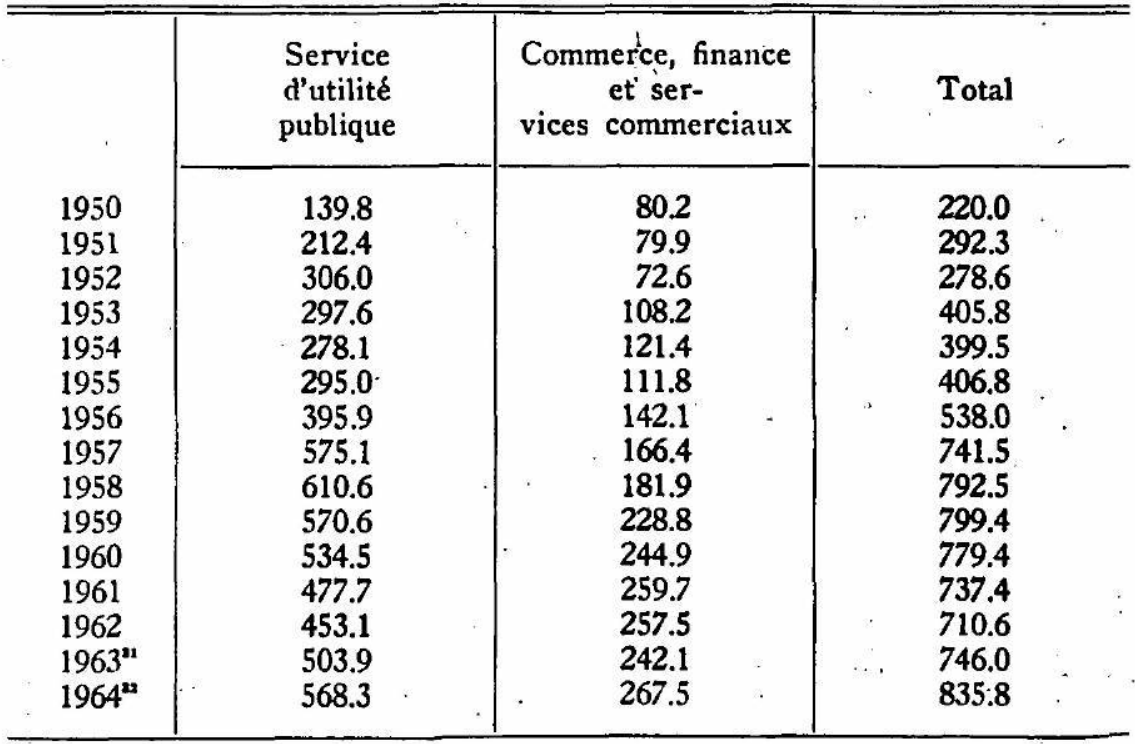

Tableau XIII

Main-d'œuvre dans le secteur tertiaire, Québec, 1950 et 1961 ss

\begin{tabular}{|c|c|c|c|c|}
\hline & \multicolumn{2}{|c|}{1951} & \multicolumn{2}{|c|}{1961} \\
\hline$\cdot$ & Unités & P.c. & Unités & P.c. \\
\hline $\begin{array}{l}\text { Administrateurs } \ldots \ldots \ldots \ldots \ldots \ldots \\
\text { Professionnels et techniciens } \ldots \ldots \\
\text { Employés de bureau } \ldots \ldots \ldots \ldots \ldots \\
\text { Vendeurs } \ldots \ldots \ldots \ldots \ldots \ldots \ldots \ldots \\
\text { Travailleurs des services } \ldots \ldots \ldots \ldots \\
\text { Travailleurs des transports } \ldots \ldots \ldots\end{array}$ & $\begin{array}{r}110,156 \\
114,176 \\
150,796 \\
77,180 \\
137,295 \\
96,199\end{array}$ & $\begin{array}{r}7.6 \\
7.9 \\
10.4 \\
5.3 \\
9.3 \\
6.7\end{array}$ & $\begin{array}{l}138,083 \\
179,705 \\
220,481 \\
105,118 \\
197,671 \\
114,665\end{array}$ & $\begin{array}{r}8.1 \\
10.4 \\
12.8 \\
6.1 \\
11.5 \\
6.7\end{array}$ \\
\hline TOTAL $\ldots \ldots \ldots \ldots \ldots \ldots \ldots \ldots \ldots \ldots$ & $.685,802$ & 47.2 & 955,723 & 55.6 \\
\hline
\end{tabular}

30. Investissements privés et publics au Canada, op. cit.

31. Chiffres préliminaires.

32. Prévisions.

33. Recensement du Canada (r95 I et r96i) O.F.S., Ottawa. 
LACTUALITE ECONOMIQUE

\begin{tabular}{|c|c|c|c|c|c|c|c|c|c|c|c|}
\hline$\ddot{\Xi}$ & $\begin{array}{l}\text { ¿ } \\
\stackrel{2}{2}\end{array}$ & ָొ & $\stackrel{\infty}{=}$ & 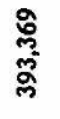 & $\stackrel{\stackrel{\circ}{\circ}}{\stackrel{7}{*}}$ & 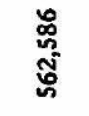 & $\begin{array}{l}\text { 莣 } \\
\text { N }\end{array}$ & 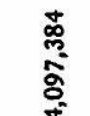 & 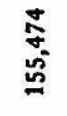 & 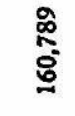 & 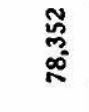 \\
\hline 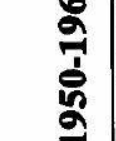 & ฏ & 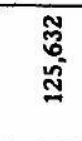 & 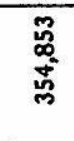 & 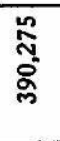 & 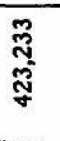 & 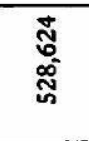 & 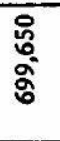 & 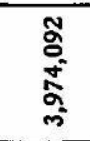 & 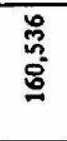 & 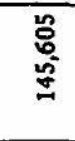 & $\begin{array}{l}\frac{0}{0} \\
\frac{0}{0}\end{array}$ \\
\hline 駕 & ڤ్ & $\stackrel{\Xi}{\Xi}$ & 总 & 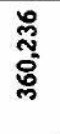 & 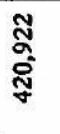 & 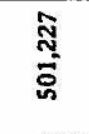 & $\begin{array}{l}\text { o } \\
0 \\
0 \\
0 \\
0\end{array}$ & 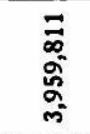 & 宮 & & 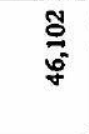 \\
\hline 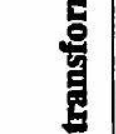 & $\underline{\tilde{a}}$ & 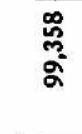 & 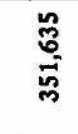 & $\begin{array}{l}\text { o } \\
\text { 产 } \\
\bar{j}\end{array}$ & 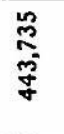 & 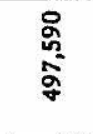 & 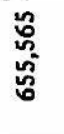 & 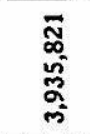 & $\begin{array}{l}\hat{a} \\
\stackrel{2}{2}\end{array}$ & 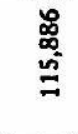 & $\begin{array}{l}\text { ôे } \\
\text { : } \\
\text { ") }\end{array}$ \\
\hline $\begin{array}{l}\stackrel{0}{0} \\
.8\end{array}$ & ڤ̊ & 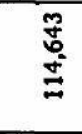 & 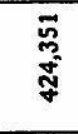 & 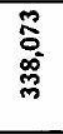 & 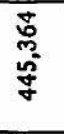 & 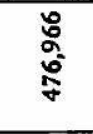 & $\begin{array}{c}\stackrel{0}{\circ} \\
\text { 今. } \\
0\end{array}$ & 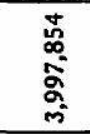 & ò & 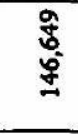 & $\begin{array}{l}\infty \\
\begin{array}{c}\infty \\
0 \\
\tilde{j}\end{array}\end{array}$ \\
\hline 兽 & 怘 & $\frac{\widetilde{o}}{+\infty}$ & 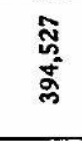 & $\begin{array}{l}\stackrel{0}{2} \\
\stackrel{\infty}{-\infty}\end{array}$ & 会 & $\begin{array}{l}\stackrel{0}{\circ} \\
\frac{a}{5}\end{array}$ & 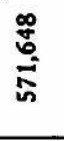 & 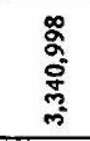 & 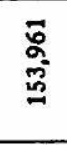 & 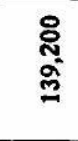 & $\begin{array}{l}\vec{D} \\
\infty \\
\vec{m}\end{array}$ \\
\hline 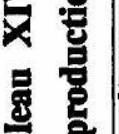 & 芯 & 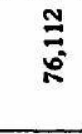 & 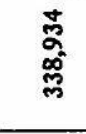 & 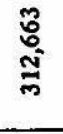 & 雇 & $\begin{array}{l}\text { \% } \\
\text { \%े } \\
\text { ळ్ }\end{array}$ & 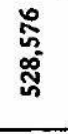 & 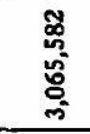 & 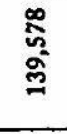 & $\begin{array}{l}\overrightarrow{\text { Z }} \\
\text { s. }\end{array}$ & $\begin{array}{l}\text { స్్ } \\
\text { నิ }\end{array}$ \\
\hline $\begin{array}{l}0 \\
8 \\
0\end{array}$ & $\stackrel{\mathscr{0}}{2}$ & $\begin{array}{l}\text { ષ. } \\
\text { م. }\end{array}$ & 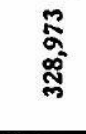 & $\begin{array}{l}\hat{े} \\
\text { 今े }\end{array}$ & $\begin{array}{l}\text { 总 } \\
\text { : } \\
\text { : } \\
\text { : }\end{array}$ & 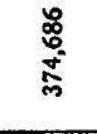 & 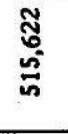 & $\begin{array}{l}\text { जै } \\
0.0 \\
0.0 \\
0 \\
0\end{array}$ & $\stackrel{\frac{m}{m}}{\frac{m}{0}}$ & 苂 & $\frac{n}{\frac{N}{5}}$ \\
\hline 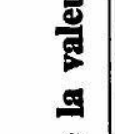 & ్ㅡㅇ & సે & 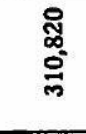 & $\begin{array}{l}\frac{\infty}{+} \\
\stackrel{\infty}{\infty} \\
\stackrel{N}{N}\end{array}$ & 商 & 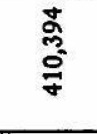 & 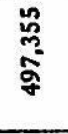 & $\begin{array}{l}\text { 今. } \\
\text { dू. } \\
\text { ה. }\end{array}$ & $\begin{array}{l}\text { ô } \\
\text { o. } \\
\text { on }\end{array}$ & $\begin{array}{l}\text { ŏ } \\
\text { oे }\end{array}$ & $\begin{array}{l}\overline{0} \\
\stackrel{-}{-}\end{array}$ \\
\hline 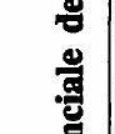 & ๘ & $\frac{1}{8}$ & 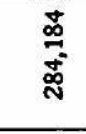 & 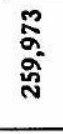 & 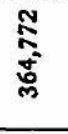 & 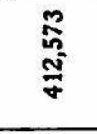 & 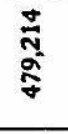 & 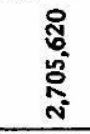 & 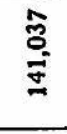 & $\frac{5}{\frac{5}{20}}$ & $\begin{array}{l}\infty \\
\stackrel{0}{\circ} \\
\text { के }\end{array}$ \\
\hline 总 & ్ㅡㅁ & 。̊ & 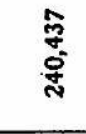 & $\begin{array}{l}\hat{0} \\
\stackrel{2}{a}\end{array}$ & $\begin{array}{l}\overrightarrow{0} \\
\text { స్ } \\
\text { స్ }\end{array}$ & $\begin{array}{l}\text { : } \\
\text { : } \\
\text { :्ల }\end{array}$ & \begin{tabular}{l}
$\stackrel{8}{8}$ \\
\multirow{5}{5}{}
\end{tabular} & 商 & $\stackrel{ \pm}{\Xi}$ & 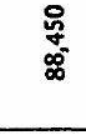 & $\begin{array}{l}\overrightarrow{5} \\
\stackrel{+}{*}\end{array}$ \\
\hline 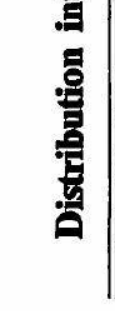 & & 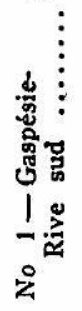 & 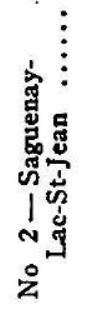 & 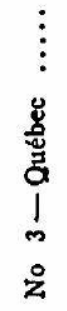 & 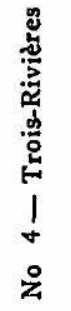 & 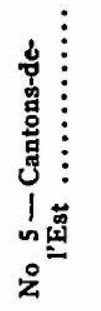 & 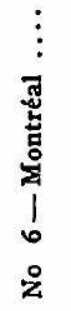 & 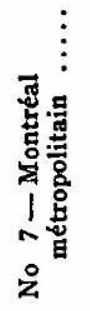 & 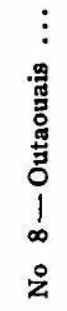 & 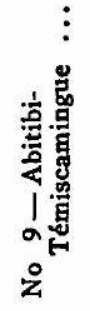 & 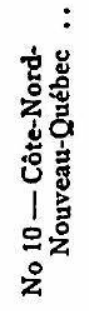 \\
\hline
\end{tabular}


EVOLUTION ECONOMIQUE DU QUEBEC

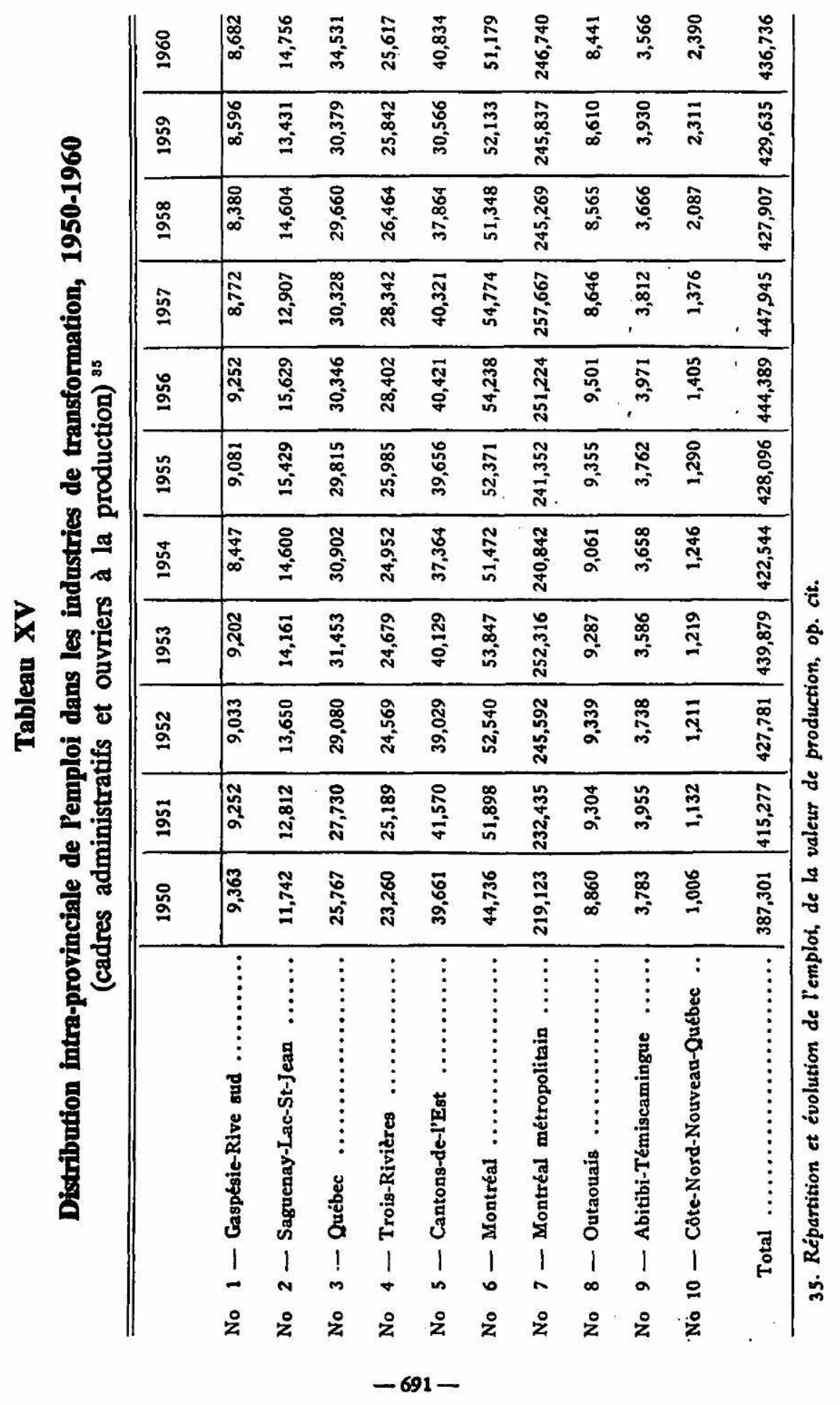




\section{L'ACTUALITE ECONOMIQUE}

\section{Tableau XVI}

Valeur de la production agricole

par région économique et par acre, $19611^{\text {so }}$

\begin{tabular}{|c|c|c|c|}
\hline & $\begin{array}{c}\text { Valeur } \\
\text { (en dollars) }\end{array}$ & $\begin{array}{c}\text { Superficie } \\
\text { (acres) }\end{array}$ & $\begin{array}{c}\text { Valeur } \\
\text { par acre } \\
\text { (en dollars) }\end{array}$ \\
\hline No 1 - Gaspésie-Rive sud & $29,539,000$ & $2,396,941$ & 12.32 \\
\hline No 2 - Saguenay-Lac-St-Jean & $12,368,000$ & 771,070 & 16.04 \\
\hline No 3 -Québec .............. & $47,714,000$ & $2,193,874$ & 21.39 \\
\hline No 4 - Trois-Rivières ... & $26,910,000$ & $1,034,888$ & 26.00 \\
\hline No 5 - Cantons-de-l'Est .. & $59,103,000$ & $2,937,049$ & 20.12 \\
\hline No 6 - Montréal ........ & $94,670,000$ & $2,975,842$ & 31.81 \\
\hline No 7 - Montréal métropolitain & $4,958,000$ & 84,685 & 58.55 \\
\hline No 8 - Outaouais $\ldots \ldots \ldots \ldots$. & $9,748,000$ & 887,961 & 10.98 \\
\hline No 9 - Abitibi-Témiscamingue ....... & $7,977,000$ & 884,896 & 9.01 \\
\hline No 10 - Côte-Nord-Nouveau-Québec .. & 363,000 & 41,286 & 8.80 \\
\hline
\end{tabular}

36. Daneau, Marcel, 《Utilisation économique des terres agricoles du Québec », Agriculture, vol. XXI, no r, janviex février 1964. 